\title{
Effect of polarized optical injection on the wavelength polarization switching and bistability of a 1550nm-VCSEL
}

\author{
A. Hurtado, I.D. Henning and M.J. Adams \\ Department of Computing and Electronic Systems, University of Essex, Wivenhoe Park, CO4 3SQ, Colchester (United Kingdom) \\ ahurt@essex.ac.uk
}

\begin{abstract}
We report the first experimental observation of a variety of forms of wavelength polarization switching and bistability with a 1550-nm VCSEL subject to optical injection with different input polarization angles.
\end{abstract}

\section{Introduction}

Bistable laser diodes and amplifiers are expected to be key components for all-optical signal processing. VerticalCavity Surface Emitting Lasers (VCSELs) are very interesting devices due to their inherent advantages, including reduced manufacturing costs, high coupling efficiency to optical fibres, ease of fabrication of $2 \mathrm{D}$ arrays etc. Therefore, the study of optical bistability $(\mathrm{OB})$ and nonlinear switching in these devices has recently undergone considerable research [1-4] and frequency polarization bistability has been reported in short-wavelength [2][3] as well as in long-wavelength VCSELs [4] subject to orthogonal optical injection. However, the effect of different polarized optical injection has as yet not received attention. In the present contribution, we report the experimental observation of wavelength polarization switching and bistability in a 1550nm-VCSEL subject to polarized optical injection into the subsidiary orthogonal polarisation mode of the device with different input polarization angles. For the first time to our knowledge a variety of nonlinear responses, including clockwise and anticlockwise wavelength polarization switching and bistability have been experimentally observed depending on the polarisation angle of the externally injected signal. This diversity of behaviour at the telecom wavelength of $1550 \mathrm{~nm}$ offers promise for the use of VCSELs in optical parallel signal processing and optical switching/routing applications.

\section{Experimental Results}

A complete description of the experimental setup can be found in [5]. The device used in the experiments was a commercially available $1550 \mathrm{~nm}$-VCSEL with a threshold current of approximately $2 \mathrm{~mA}$ at $298^{\circ} \mathrm{K}$. The spectrum of the device exhibited two modes corresponding to the two orthogonal polarizations of the fundamental mode. The lasing mode of the VCSEL has parallel polarization $\left(0^{\circ}\right)$ and is located at $1543.2 \mathrm{~nm}$, while the second subsidiary mode has orthogonal polarization $\left(90^{\circ}\right)$ and is shifted approximately $0.5 \mathrm{~nm}$ to the long wavelength side of the lasing mode $\left(\lambda_{\text {VCSEL }}=1543.7 \mathrm{~nm}\right)$. The polarization of the VCSEL was stable and no polarization switching was experimentally observed for any bias above threshold. The measurements were taken with the device biased with an applied current of $3 \mathrm{~mA}\left(\mathrm{I} / \mathrm{I}_{\mathrm{th}} \approx 1.5\right)$ and at a constant temperature of $298{ }^{\circ} \mathrm{K}$. An external optical signal with a constant input power of $500 \mu \mathrm{W}$ was injected into the subsidiary orthogonal polarized mode of the device.

Figs. 1(a) and 1(b) show the measured reflected optical power for the parallel and the orthogonal polarization modes of the VCSEL as a function of the initial wavelength detuning for the case of orthogonal optical injection (input polarisation angle of $90^{\circ}$ ). As it has been previously reported [2-4], two consecutive polarisation switching points occur as the wavelength of the external signal is scanned from negative to positive wavelength detuning. The first one, in the negative wavelength detuning region, appears as a consequence of the locking of the subsidiary orthogonal mode of the device to the externally injected signal. The output of the parallel-polarized mode is then suppressed and the polarization at the output of the device switches from
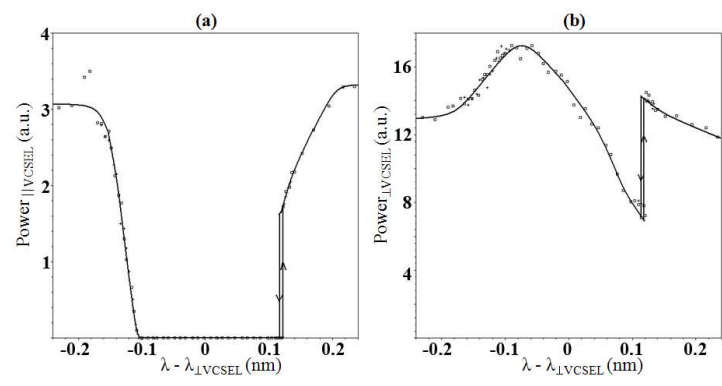

Fig. 1. Reflected output power vs. initial wavelength detuning (a) for the parallel polarized emitting mode and (b) for the orthogonal polarized mode of the $1550 \mathrm{~nm}$-VCSEL subject to orthogonallypolarized optical injection (input polarization angle of $90^{\circ}$ ).

parallel to orthogonal. The second switching point, appearing in the positive wavelength detuning region, shows the reverse process and occurs when the orthogonal mode of the VCSEL becomes unlocked from the externally injected signal. Switching from orthogonal to parallel polarization is now produced. As a result, the form of the detuning 
curve for the parallel polarized mode is similar to that reported in [2-4], and as in [4] anticlockwise wavelength bistability is experimentally measured for the output of the orthogonal polarized mode associated with the second polarization switching point.

Figs. 2(a-d) and 2(e-g) show respectively the measured reflected optical power for the orthogonal and the parallel polarised mode of the VCSEL for four different intermediate values of the input polarisation angle of the externally injected signal, namely $60^{\circ}, 45^{\circ}, 30$ and $15^{\circ}$. For both polarization modes of the device, the more the input polarisation angle of the externally injected signal approaches $90^{\circ}$, the more asymmetrical are the experimentally measured curves and the switching becomes more abrupt until bistability is observed. For the output of the parallel polarized mode of the device (figs. 2(e-h)) the characteristic nonlinear switching or hysteresis curve appears in all cases. On the other hand, and for the first time to our knowledge, a variety of nonlinear responses have been experimentally observed for the output of the orthogonal polarized mode as the input polarization angle of the external signal is modified. Initially, for an input polarization angle of $60^{\circ}$, still close enough to the polarization of the mode subject to injection, anticlockwise wavelength bistability is observed (see fig. 2(a)) as in the case of orthogonal optical injection included in fig. 1(b). Then, as the input polarization angle is reduced, the on-off contrast ratio between output states is decreased until a change in the shape in the nonlinear transition is produced from the anticlockwise wavelength switching appearing for input polarisation angles of $60^{\circ}$ and $45^{\circ}$ (figs. 2(a) and 2(b)), to the occurrence of clockwise wavelength switching for input polarisation angles of $30^{\circ}$ and $15^{\circ}$ (figs. 2(c) and 2(d)).

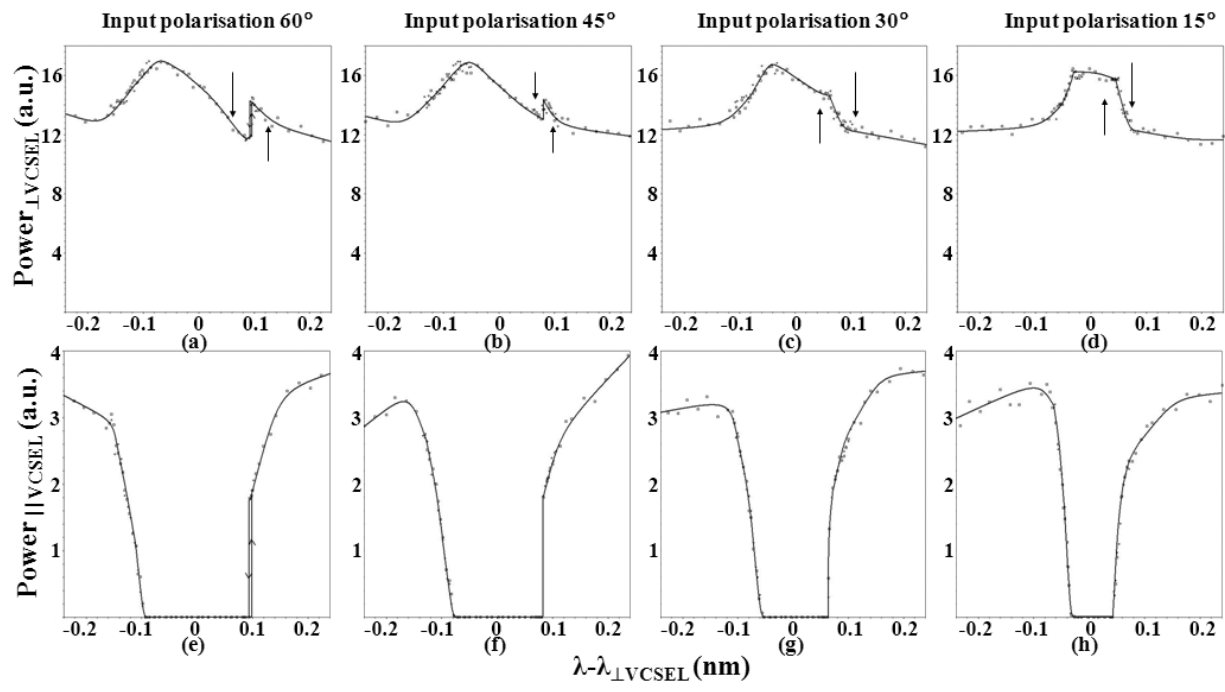

Fig. 2. Reflected output power vs. initial wavelength detuning for the orthogonal (a-d) and the parallel (e-h) polarized emitting mode of the $1550 \mathrm{~nm}-\mathrm{VCSEL}$ for four different input polarization angles of the optically injected signal, namely $60^{\circ}(\mathrm{a}-\mathrm{e}), 45^{\circ}(\mathrm{b}-\mathrm{f}), 30^{\circ}(\mathrm{c}-\mathrm{g})$ and $15^{\circ}(\mathrm{d}-\mathrm{h})$.

\section{Conclusions}

We report a first experimental observation of polarization wavelength switching and bistability in a 1550nm-VCSEL under different cases of polarized optical injection into the orthogonally-polarized subsidiary mode of the device. The output of both polarization modes has been analyzed experimentally and a variety of nonlinear responses including clockwise and anticlockwise as well as polarization wavelength switching and bistability has been observed depending on the input polarization angle of the externally injected signal. This diversity of behavior in a VCSEL at the important telecom wavelength of $1550 \mathrm{~nm}$ offers promise for the use of these devices in optical signal processing and optical switching/routing applications.

\section{References}

1. B.S. Ryvkin, K. Panajotov, E.A. Avrutin, I. Veretennicoff and H. Thienpont, J. Appl. Phys. 96, 6002 (2004)

2. Y. Hong, K.A. Shore, A. Larsson, M. Ghisoni and J. Halonen, Electron. Lett. 36, 2019 (2000)

3. I. Gatare, J. Buesa, H. Thienpont, K. Panajotov and M. Sciamanna, Phys. Rev. A 75, 023804 (2007)

4. A. Hurtado, I.D. Henning and M.J. Adams, CLEO 2008, San José, CA, USA, (2008)

5. A. Hurtado, I.D. Henning and M.J. Adams, IEEE J. Sel. Topics Quantum Electron. 14, 911 (2008) 